\title{
ArTícUlO
}

\section{Familia Parapaguridae (Anomura, Paguroidea) en Chile: Nuevos registros de cangrejos ermitaños de profundidad}

\author{
The family Parapaguridae (Anomura, Paguroidea) in Chile: New records from deep water hermit crabs
}

\section{Nicole Olguín ${ }^{1}$, Guillermo Guzmán ${ }^{1,2}$ y Eduardo Quiroga ${ }^{3}$}

\author{
${ }^{1}$ Laboratório de Carcinologia, Museu de Zoologia, Universidade de São Paulo, CEP 04263-000, São Paulo, SP, Brasil. \\ nolguincamp@yahoo.es \\ ${ }^{2}$ Museo del Mar, Universidad Arturo Prat, Casilla 121, Iquique, Chile \\ ${ }^{3}$ Escuela de Ciencias del Mar, Pontificia Universidad Católica de Valparaíso, Av. Altamirano \#1480, Valparaíso, Chile
}

\begin{abstract}
Two new distribution records of hermit crabs of the family Parapaguridae, i.e., Oncopagurus haigae and Sympagurus dimorphus are presented. The material examined was collected by 2 expeditions from the Chilean coast. The PUCK-156 and INSPIRE Expeditions in 2001 and 2010, respectively, at depths ranging from 340 to $1300 \mathrm{~m}$. The present study provides taxonomic and distributional information as well as a key to aid in the identification of the species of Parapaguridae present in waters off Chile. With these results the family in southeast Pacific waters off Chile is represented by 14 species belonging to the genera Oncopagurus (3 species), Paragiopagurus (3 species), Parapagurus (3 species), Sympagurus (3 species), Strobopagurus (one species) and Tylaspis (one species).
\end{abstract}

Key words: Parapaguridae, deep-water hermit crab, distribution, Chile

Resumen.- Se dan a conocer 2 nuevos registros de cangrejos ermitaños de la familia Parapaguridae, Oncopagurus haigae y Sympagurus dimorphus. El material examinado fue recolectado por 2 expediciones frente a las costas de Chile. La expedición PUCK-156 y la expedición INSPIRE 2010 realizadas en los años 2001 y 2010, respectivamente, a profundidades entre los 340 a $1300 \mathrm{~m}$. El presente estudio proporciona información taxonómica y de distribución, así como, una clave para ayudar en la identificación de las especies de Parapaguridae presentes en aguas frente a Chile. Con estos resultados, la familia en aguas del Pacífico sureste frente a Chile, queda representada por 14 especies pertenecientes a los géneros Oncopagurus (3 especies), Paragiopagurus (3 especies), Parapagurus (3 especies), Sympagurus (3 especies), Strobopagurus (una especie) y Tylaspis (una especie).

Palabras clave: Parapaguridae, aguas profundas, distribución geográfica, Chile

\section{INTRODUCCIÓN}

La familia Parapaguridae Smith, 1882, corresponde a un taxón cosmopolita de cangrejos ermitaños, distribuidos en aguas profundas en la mayoría de los océanos, siendo encontrados principalmente entre los $100 \mathrm{~m}$ y hasta más de $4000 \mathrm{~m}$ de profundidad (Menzies et al. 1973, Lemaitre 1986, 1989; Cardoso \& Lemaitre 2012). Los reportes de estas especies no han sido muy frecuentes debido a la dificultad de obtener muestras biológicas en profundidades mayores a $500 \mathrm{~m}$. Para Chile, la mayoría de los registros corresponden a citas únicas (e.g., Parapagurus janetae Lemaitre, 1999, Parapagurus holthuisi Lemaitre, 1989).

En la actualidad, la familia Parapaguridae está constituida por 10 géneros (McLaughlin et al. 2010), de los cuales 6 están representados en aguas frente a Chile, incluyendo las especies citadas para las cercanías de los montes submarinos de la placa de Nazca, con un total de 14 especies: 3 pertenecen al género Oncopagurus Lemaitre, 1996, 3 a Parapagurus Smith, 1879, 3 a Sympagurus Smith, 1883, 3 a Paragiopagurus Lemaitre, 1996 y los géneros Strobopagurus Lemaitre, 1989 y Tylaspis Henderson, 1885 con un representante cada uno (Haig 1955, Lemaitre \& McLaughlin 1992, Zhadan 1997, Lemaitre 1999).

Los primeros antecedentes sobre la familia Parapaguridae en Chile se remontan a la expedición Challenger, en la que Henderson (1888) registra un ejemplar de Sympagurus dimorphus (Studer, 1883) (como Parapagurus dimorphus) en Puerto Churruca $\left(\sim 52^{\circ} \mathrm{S}\right)$. Otros registros de $S$. dimorphus para Chile corresponden al material recolectado por el R/V Eltanin, en el Cabo de Hornos $\left(\sim 56^{\circ} \mathrm{S}\right)$ y frente a la península de Taitao $\left(\sim 46^{\circ} \mathrm{S}\right)$ 
entre los años 1964 a 1966 (Lemaitre 1989, Lemaitre \& McLaughlin 1992). Además en la misma expedición, se recolectaron 6 ejemplares los que fueron asignados a Parapagurus abyssorum Henderson, 1888 [Homonimia de Parapagurus abyssorum (Filhol, 1885)]. Sin embargo posteriores revisiones revelaron que esos ejemplares corresponden a las especies Parapagurus holthuisi Lemaitre, 1989 frente a Valparaíso $\left(\sim 33^{\circ} \mathrm{S}\right)$ y a P. janetae, en Puerto Otway $\left(\sim 46^{\circ} S\right)$ (Lemaitre 1989, 1999). Por otra parte, entre las muestras obtenidas por esta expedición, se obtuvieron 2 ejemplares de Parapagurus abyssorum (Filhol, 1885) (Lemaitre 1999), los que, en sentido estricto, corresponden a la única cita correcta de esta especie para aguas frente a Chile.

Con posterioridad, Porter (1906) en un trabajo acerca de los crustáceos de la zona de Los Vilos $\left(\sim 31^{\circ} \mathrm{S}\right)$ cita a Parapagurus pilosimanus Smith, 1879. Según Haig (1955) correspondería a un error de identificación dado que el material provendría de aguas someras. Desafortunadamente, en el trabajo de Porter no se hace mención de la procedencia exacta del material.

Oncopagurus haigae (de Saint Laurent, 1972), corresponde a otra de las especies citadas para Chile. Descrita originalmente frente a Isla Santa Cruz California (de Saint Laurent 1972, Wicksten 1987), fue luego registrada frente a Valparaíso por Lemaitre \& McLaughlin (1992) como S. haigae. Esta especie ha sufrido varios cambios en su estatus genérico, fue descrita inicialmente como miembro de Parapagurus por de Saint Laurent (1972). Posteriormente, fue asignada por Lemaitre (1989) al género Sympagurus Smith, 1883. Finalmente Lemaitre (1996) describe el género Oncopagurus, argumentando la reubicación de esta especie en este taxòn.

Otro importante aporte al conocimiento de la diversidad de la familia Parapaguridae, fue la realización de diversas expediciones de la ex Unión Soviética en los alrededores de los montes submarinos de la placa de Nazca, a bordo de los B/C Ikhtiandr, Professor Mesyatsev y Professor Stokman, entre 1973 y 1987 (Parin et al. 1997). Durante estas expediciones, se registraron 10 especies, siendo algunos de estos registros los únicos antecedentes existentes de su presencia en aguas frente a Chile (Zhadan 1997).

Entre los años 2001 y 2010, 2 expediciones científicas a lo largo del margen continental chileno, en asociación con instituciones chilenas y extranjeras han permitido recolectar cangrejos ermitaños pertenecientes a la familia Parapaguridae (Sellanes et al. 2007) ${ }^{1}$. En el presente trabajo se realiza una revisión de parte de estos muestreos, con el objetivo de dar a conocer nuevos registros de especies de cangrejos ermitaños en el margen continental de Chile, recopilar información del grupo y entregar una clave para la identificación taxonómica de la familia Parapaguridae en aguas frente a Chile.

\section{Materiales Y MÉTODOS}

El material analizado proviene de 2 expediciones en aguas chilenas; (i) la expedición PUCK-156, realizada entre el 2 de abril al 5 de mayo, 2001, frente a Antofagasta $\left(\sim 22^{\circ} \mathrm{S}\right)$, Concepción $\left(\sim 36^{\circ} \mathrm{S}\right)$ y Chiloé $\left(\sim 42^{\circ} \mathrm{S}\right)$, a bordo de B/C SONNE (Hebbeln et al. 2001, Quiroga et al. 2009); y (ii) la expedición INSPIRE, a bordo del R/V Melville entre el 23 de febrero al 17 de marzo del 2010, frente a Valparaíso $\left(\sim 32^{\circ} \mathrm{S}\right)$, Concepción y frente a la Península de Taitao ( $\sim 6^{\circ} \mathrm{S}$ ) (Quiroga \& Levin 2010).

A cada ejemplar se le midió la longitud del escudo (le) que corresponde a la distancia en milímetros ( $\mathrm{mm}$ ), desde el punto medio del lóbulo rostral al punto medio del margen posterior del escudo. Los especímenes examinados fueron depositados en la colección de decápodos del Museo del Mar de la Universidad Arturo Prat (MUAP), Iquique, Chile. Otras abreviaciones usadas en el texto corresponden a: esp= especímenes; est= estación.

\section{Resultados}

\section{TAXonomía}

Superfamilia Paguroidea Latreille, 1802

Familia Parapaguridae Smith, 1882.

GÉNERo OnCOPAGURUs Lemaitre, 1996

OnCopagurus haigaE (de Saint Laurent, 1972) (Fig. 1)

Material examinado: Expedición PUCK-156, 40 esp, 7 esp (le = 2,93-4,62 mm), est 7163 (AGT-11) frente a Concepción (3625,49'S; 7335,72'W), 538-550 m, MUAP (CD) 0452 y 33 esp (le = 3,41-5,02 mm), est 7163 (AGT-18) frente a Chiloé (42 $35,35^{\prime}$ S; $74^{\circ} 48,33^{\prime} \mathrm{W}$ ) entre los 480-502 m, MUAP (CD) 0344. Expedición INSPIRE, 6 esp, 2 esp (1 $0^{\circ}$ le $=2,87 \mathrm{~mm}$ y 1 q le = 4,62 mm), est 27 (AGT-2), frente a

${ }^{1}$ Sellanes J, E Quiroga \& C Neira. 2007. Megafauna bentónica asociada a los Sistemas de Filtración de Metano frente a Chile Central ( $33^{\circ} 23^{\prime}$ a $37^{\circ} 57^{\prime}$ S). Reporte Crucero VG07-Pata I, Valparaíso-Talcahuano (27 septiembre-06 octubre, 2007). 
Península de Taitao (4654,155'S, 75³5,997’ W) entre 474497 m, MUAP (CD) 0453; 1 esp (o le = 5,06 mm), est 66

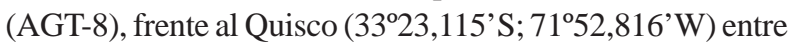
344-382 m, MUAP (CD) 0454, y 3 esp (3 o' le=3,37-4,53 $\mathrm{mm}$ ), est 68 (AGT-10) frente a El Quisco (3322,937's; 7152,780’W) entre 337-343 m, MUAP (CD) 0455.

Diagnosis: Escudo cefálico tan largo como ancho (Fig. 1a). Acículo ocular simple, escafocerito multidentado (Fig. 1a). Quelípedo derecho armado con espinas cortas y densamente cubiertos de cerdas (Fig. 1b). Superficie dorsal del própodo del quelípodo izquierdo desarmado o con pocas espinas pequeñas (Fig. 1c). Margen dorsal del carpo del segundo pereiópodo con una espina distal (Fig. 1d). Escofina propodal del cuarto pereiópodo con 1 fila de escamas córneas en todo el margen ventral y con 2 filas en la porción proximal (Fig. 1e). Telson con 5 espinas en el lóbulo mayor y 4 en el lóbulo menor en los machos, y variable en las hembras (Fig. 1f). Espina epistomial curva hacia arriba (Fig. 1g). Sin pleurobranquia en el octavo somito torácico (sobre el quinto par de pereiópodos).

Distribución geográfica: Desde el Golfo de California a Valparaíso (de Saint Laurent 1972, Lemaitre \& McLaughlin 1992). Montes submarinos de la placa de Nazca (Zhadan 1997). En Chile desde los $33^{\circ} \mathrm{S}$ a los $43^{\circ} \mathrm{S}$.

Comentarios: Durante el octavo crucero de investigaciones marinas de áreas remotas (CIMAR) del Comité Oceanográfico Nacional, se recolectaron algunos ejemplares de Parapaguridae asignados a Oncopagurus sp. (Retamal 2007). Algunos de esos ejemplares fueron enviados al instituto Smithsoniano, quedando catalogados posteriormente como O. haigae (USNM 1008981). El material fue recolectado en la estación número 4 de ese crucero a 189 m de profundidad en la boca del Guafo, frente al golfo del Corcovado en la XI Región de Chile (43³9,36’'s; $\left.73^{\circ} 51,11^{\prime} \mathrm{W}\right)$.

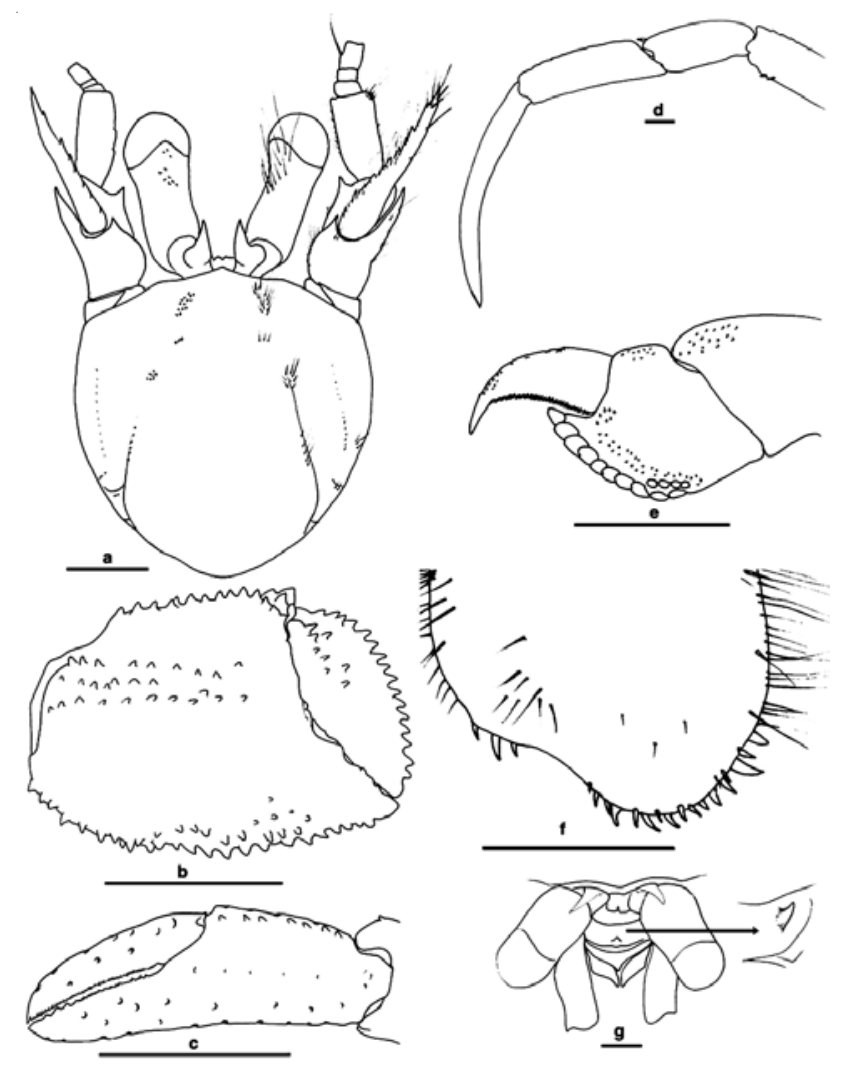

Figura 1. Oncopagurus haigae (de Saint Laurent, 1972). Hembra (MUAP(CD)-0454, le= 5,06 mm): a) escudo y apéndices cefálicos, b) quelípodo derecho (cerdas omitidas), c) quelípodo izquierdo (cerdas omitidas), d) segundo pereiópodo, e) própodo y dáctilo del cuarto pereiópodo, f) telson, $\mathrm{g}$ ) diagrama de la región anterior del escudo mostrando la espina epistomial. Barra de escalas= $1 \mathrm{~mm}(\mathrm{a}-\mathrm{d}, \mathrm{f}-\mathrm{g})$ y 0,5 mm (e) / Oncopagurus haigae (de Saint Laurent, 1972). Female (MUAP(CD)-0454, le $=5.06 \mathrm{~mm}$ ): a) shield and cephalic appendages, b) right cheliped (setae omitted), c) left cheliped (setae omitted), d) second pereopod, e) propodus and dactyl of fourth pereopod, f) telson, g) diagram of the anterior region of shield, showing the epistomial spine. Scale bars equal $1 \mathrm{~mm}$ (a-d, $\mathrm{f}-\mathrm{g}$ ) and $0.5 \mathrm{~mm}$ (e)

Vol. 49, № 1, 2014 
Estos nuevos registros fueron recolectados en las cercanías de una zona de emanaciones de metano frente a Concepción.

GÉNERo Sympagurus Smith, 1883

SYMPAGURUS DIMORPHUS (StUDER, 1883) (Fig. 2)

Material examinado: Expedición INSPIRE, 2 esp, 1 esp ( $1 \mathrm{O}^{\prime \prime} \mathrm{le}=7,60 \mathrm{~mm}$ ), est 26 (AGT-1) frente a la Península de Taitao (4655,216S, 75³4,852’W), 460-697 m, MUAP(CD) 0456; 1 esp ( $1 \mathrm{O}^{\mathrm{h}} \mathrm{le}=3,21 \mathrm{~mm}$ ), est 42 (AGT-3), frente a Talcahuano (36²2,523’S, 7343,114'W) 724 a 764 m, MUAP (CD) 0457.
Diagnosis: Escudo dorsal levemente más ancho que largo. Acículo ocular simple, escafocerito multiespinoso (Fig. 2a). Quelípodo derecho 3 veces mayor que el izquierdo y densamente piloso (Fig. 2b). Quelípodo izquierdo delgado, desarmado o con pequeños tubérculos y espinas (Fig. 2c). Espina epistomial pequeña, recta. Margen dorsal del carpo del tercer pereiópodo con una fila de espinas (Fig. 2d). Escofina propodal del cuarto pereiópodo con 2 filas de escamas córneas (Fig. 2e). Telson más o menos recto con una suave concavidad media, márgenes espinosos (Fig. 2f). Rudimento de pleurobranquia en el octavo segmento torácico (Fig. 2g).

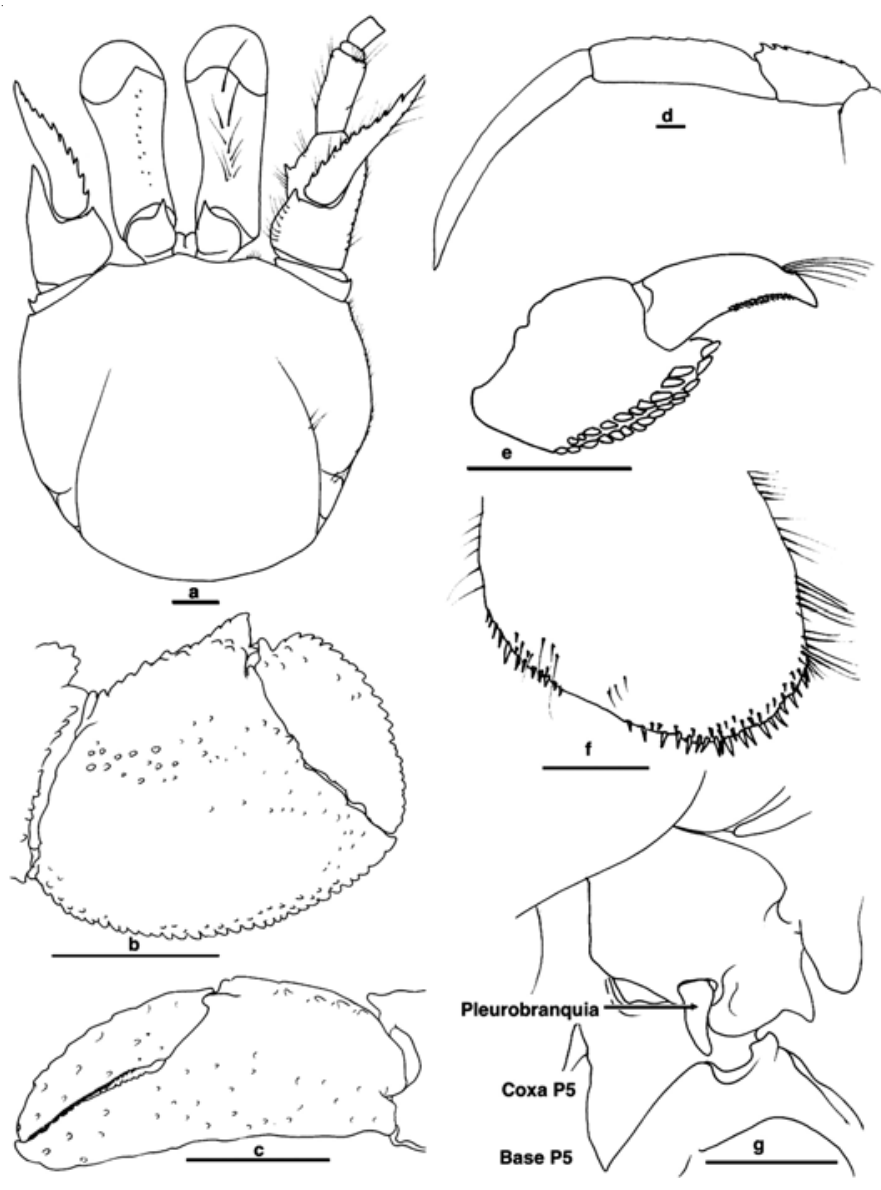

Figura 2. Sympagurus dimorphus (Studer, 1883). Macho (MUAP(CD)-0456, le= 7,60 mm): a) escudo y apéndices cefálicos, b) quelípodo derecho (cerdas omitidas), c) quelípodo izquierdo (cerdas omitidas), d) segundo pereiópodo, e) própodo y dáctilo del cuarto pereiópodo (cerdas omitidas), f) telson, g) diagrama de último segmento torácico, mostrando la pleurobranquia vestigial. Barra de escalas= $1 \mathrm{~mm} /$ Sympagurus dimorphus (Studer, 1883) Male (MUAP(CD)-0456, le= $7.60 \mathrm{~mm}$ ): a) shield and cephalic appendages, b) right cheliped (setae omitted), c) left cheliped (setae omitted), d) second pereopod, e) propodus and dactyl of fourth pereopod (setae omitted), f) telson, g) diagram of the last thoracic somite, showing of the vestigial pleurobranchia. Scale bars $=1 \mathrm{~mm}$ 
Distribución geográfica: Ampliamente distribuido en el hemisferio sur, Australia, Sudáfrica e Islas Marion. Estrecho de Magallanes, Chile (Henderson 1888, Haig 1955, Lemaitre 1989, 2004).

Comentarios: Citado para el extremo sur de Chile como Parapagurus dimorphus por Henderson (1888). Posteriormente fue registrada frente a Chile durante la expedición Eltanin, a más de 1100 km de la costa (Lemaitre 1989, Lemaitre \& McLaughlin 1992). Este es un nuevo registro y nueva localidad frente a Talcahuano $\left(\sim 36^{\circ} \mathrm{S}\right)$, extendiendo su distribución hacia el norte en más de 1000 km del anterior registro para Chile. Los ejemplares fueron encontrados asociados a un zoanthideo, sobre la concha del gastrópodo Fusitriton magellanicus (Röding 1798).

\section{Otros REgistros de LA FAMILIA EN AgUAS FRENTE A CHILE}

En la Tabla 1 se entrega una lista de otras especies de esta familia citadas en la literatura para aguas del Pacífico Sureste con sus respectivas referencias.

Tabla 1. Lista de especies de Parapaguridae, en aguas Chilenas, incluyendo los montes submarinos de la placa de Nazca, con la respectiva localidad y referencias del registro / List of species of Parapaguridae, in Chilean waters, including the submarine ridges of Nazca plate, with respective locality of collection and references

\begin{tabular}{|c|c|c|}
\hline Especie & Localidad & Referencia \\
\hline Oncopagurus mironovi Zhadan, 1997 & Montes submarinos de la placa de Nazca & Zhadan 1997 \\
\hline Oncopagurus stockmani Zhadan, 1997 & Montes submarinos de la placa de Nazca & Zhadan 1997 \\
\hline Oncopagurus haigae (de Saint Laurent, 1972) & $\begin{array}{l}\text { Valparaíso } \\
\text { Montes submarinos de la placa de Nazca } \\
\text { El Quisco a Península de Taitao }\end{array}$ & $\begin{array}{l}\text { Lemaitre \& McLaughlin } 1992 \\
\text { Zhadan } 1997 \\
\text { Este reporte }\end{array}$ \\
\hline Parapagurus abyssorum (Filhol, 1885) & Frente a Valdivia $1930 \mathrm{Km}$ & Lemaitre 1999 \\
\hline Parapagurus holthuisi Lemaitre, 1989 & Archipiélago de Juan Fernández & Lemaitre 1999 \\
\hline Parapagurus janetae Lemaitre, 1999 & Puerto Otway, Golfo de Penas & Lemaitre 1999 \\
\hline Paragiopagurus boletifer (de Saint Laurent, 1972) & Montes submarinos de la placa de Nazca & Zhadan 1997 \\
\hline Paragiopagurus ruticheles (A. Milne-Edwards, 1891) & Montes submarinos de la placa de Nazca & Zhadan 1997 \\
\hline Paragiopagurus wallisi (Lemaitre, 1994) & Montes submarinos de la placa de Nazca & Zhadan 1997 \\
\hline $\begin{array}{l}\text { Strobopagurus aff. gracilipes (A. Milne Edwards, 1891) } \\
\text { (ver nota al pie de tabla) }\end{array}$ & Montes submarinos de la placa de Nazca & Zhadan 1997 \\
\hline Sympagurus affinis (Henderson, 1888) & Montes submarinos de la placa de Nazca & Zhadan 1997 \\
\hline Sympagurus dimorphus (Studer, 1883) & $\begin{array}{l}\text { Puerto Churruca } \\
\text { Frente a Península de Taitao } \\
\text { Frente a Talcahuano }\end{array}$ & $\begin{array}{l}\text { Henderson } 1888 \\
\text { Lemaitre } 1989 \\
\text { Este reporte }\end{array}$ \\
\hline Sympagurus dofleini (Balss, 1912) & Montes submarinos de la placa de Nazca & Lemaitre 2004 \\
\hline Tylaspis anómala Henderson, 1885 & Nor-Este de Isla de Pascua & Lemaitre 1998 \\
\hline
\end{tabular}

Nota: Zhadan (1997) asigna tentativamente algunos ejemplares recolectados en los montes submarinos de la placa de Nazca, como Paragiopagurus aff. gracilipes. Sin embargo, Lemaitre (2004) sugiere que pueden corresponder a Bivalvopagurus sinensis (de Saint Laurent, 1972) o a una especie no descrita de este género. Se optó por respetar la cita original mientras no exista claridad acerca del estatus taxonómico de estos ejemplares. 
Clave para la identificación de las especies de Parapaguridae registradas en aguas Chilenas, incluyendo los montes submarinos de la placa de Nazca (Modificado y ADAPTADO DE LEMAITRE 1996, 2004)

1 Acículos oculares presentes ....

Acículos oculares ausentes. Rostro triangular prominente, alcanzando los pedúnculos oculares. Caparazón y abdomen parcialmente calcificado

Tylaspis anomala Henderson, 1885.

2(1) Escudo dorsal casi tan ancho como largo. Dáctilo de las patas ambulatorias (segundo y tercer par de pereiópodos) curvos. Córneas menos de la mitad del diámetro del pedúnculo ocular. Segundo par de pleópodos de los machos sin exópodo y extremo distal no curvado 3

Escudo dorsal más ancho que largo. Dáctilo de las patas ambulatorias rectas. Córneas fuertemente dilatadas, el doble o casi el doble del diámetro de los pedúnculos oculares. Segundo par de pleópodos de los machos con un exópodo corto y fuertemente curvado distalmente. Própodo del cuarto pereiópodo tan largo como ancho

Strobopagurus gracilipes (A. Milne-Edwards, 1891)

3(2) Último segmento torácico con una pleurobranquia vestigial (Fig. 2g) (Género

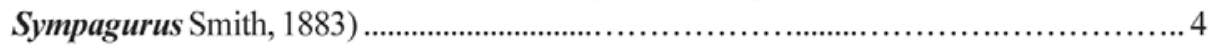

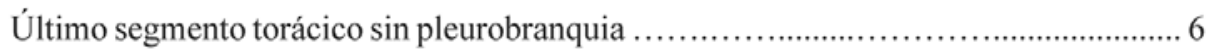

4(3) Láminas branquiales del tipo cuadriseriales profundas $\ldots \ldots \ldots \ldots \ldots \ldots \ldots \ldots \ldots \ldots$

Branquias biseriales o sólo distalmente cuadriseriales. Telson levemente asimétrico, margen terminal con espinas córneas. Escofina propodal del cuarto par de pereiópodos con 3 o 4 filas de escamas cónicas

Sympagurus dofleini (Balss, 1912)

5(4) Carpo del segundo y tercer par de pereiópodos (primer y segundo par de patas ambulatorias) con una fila de espinas en el margen dorsal. Espina epistomial presente

Sympagurus dimorphus (Studer, 1883)

Carpo de segundo y tercer par de pereiópodos (primer y segundo par de patas ambulatorias) sin espinas en margen dorsal excepto por la espina dorsodistal. Espina epistomial ausente. Dáctilos del primer y segundo par de patas ambulatorias con $10 \mathrm{o}$ más espínulas córneas en el margen ventromesial .

Sympagurus affinis (Henderson, 1888)

6(3) Espina epistomial fuertemente curvada hacia arriba (Fig. 1g) (Género Oncopagurus

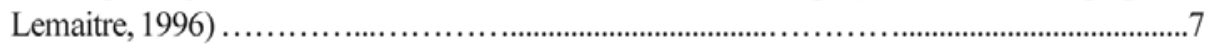

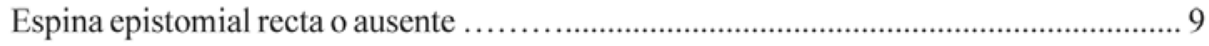

7(6) Margen dorsal de la quela menor armada de tubérculos y/o espinas. Escofina propodal del cuarto par de pereiópodos con escamas distales y proximales .............. 8

Margen dorsal de la quela menor no armada. Escofina propodal del cuarto par de pereiópodos con sólo una fila distal de escamas .

Oncopagurus mironovi Zhadan, 1997

8(7) Proyecciones laterales del caparazón con una espina. Escofina propodal del cuarto par de pereiópodos con 2 filas de escamas

Oncopagurus stockmani Zhadan, 1997

Sin espinas en los márgenes del caparazón. Escofina propodal con una fila de escamas en la porción distal Oncopagurus haigae (de Saint Laurent, 1972) 
9(6) Branquias bi o cuadriseriales. Cuarto segmento del pedúnculo antenal armado con una espina dorsal distal. (Género Paragiopagurus Lemaitre, 1996)

Branquias cuadriseriales. Cuarto segmento del pedúnculo antenal sin espina. (Género Parapagurus

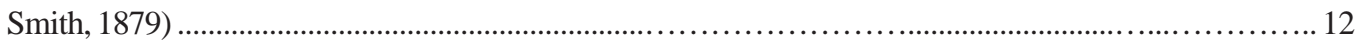

10(9) Cara ventral de la quela derecha armada con tubérculos ........................................... 11

Cara ventral de la quela derecha con hendiduras transversales que se extienden en el carpo

Paragiopagurus ruticheles (A. Milne-Edwards, 1891)

11(10) Cara ventral de la quela derecha cubierta con tubérculos prominentes en forma de hongos. Carpo de la quela armada con espinas y tubérculos dispersos .....

Paragiopagurus boletifer (de Saint Laurent, 1972)

Cara ventral de la quela derecha sin armadura, con pequeñas espinas o tubérculos. Carpo de la quela derecha desarmado ................................................... Paragiopagurus wallisi (Lemaitre, 1994)

12(9) Caras externas del própodo, mero y carpo de las patas ambulatorias (segundo y tercer par de pereiópodos) con espinas. Margen antero distal armado con pequeñas espinas. Escofina propodal del cuarto par de pereiópodos con dos o tres filas de escamas lanceoladas

Parapagurus abyssorum (Filhol, 1885)

Cara externa del própodo, mero y carpo de las patas ambulatorias sin espina ........................ 13

13(12) Largo del própodo del segundo par de patas ambulatorias hasta 4,3 veces el alto. Largo del mero del mismo apéndice a lo más 3 veces el alto .................. Parapagurus janetae Lemaitre, 1999

Largo del própodo del segundo par de patas más de 4,3 veces el alto. Largo del mero mucho más de 3 veces el alto

Parapagurus holthuisi Lemaitre, 1999

\section{Discusión}

Los reportes de la fauna de cangrejos ermitaños de la familia Parapaguridae en las costas de Chile son escasos, producto de la dificultad de obtener material biológico proveniente de aguas profundas. Esto genera una impresión, que puede ser errada, respecto de la distribución geográfica de las especies, sobre todo de las que presentan registros en puntos ampliamente separados.

Tal es así que al momento de analizar los registros se observan especies con una distribución biogeográfica amplia y continua en prácticamente todo el Pacífico este. Por otro lado, al no contar con registros en zonas intermedias de la distribución, esta presunción pudiera enmascarar una distribución anfipacífica o de otro tipo. La solución de este problema sólo puede venir de la mano de mayores esfuerzos para conocer la diversidad del grupo en particular o de la fauna de mares profundos en general.

La distribución de Oncopagurus haigae, cuya cita original fue para Isla Santa Cruz California, luego para Valparaíso y posteriormente hasta Chiloé, Chile, puede ser explicada por conexiones de las masas de aguas profundas del Pacífico, las que se registran por debajo de los $1.000 \mathrm{~m}$ de profundidad y asociada a aguas intermedias antárticas que se profundizan hacia el norte de Chile (Silva \& Konow 1975). La distribución de estos cangrejos ermitaños sigue el patrón de otros decápodos registrados en aguas profundas frente a Chile, tales como Cymonomus menziesi Garth, 1971, algunos camarones como Metacrangon procax Faxon, 1893, o especies de cangrejos braquiuros como Trichopeltarium spp., entre otros (Guzmán 2003, Guzmán \& Quiroga 2005, Guzmán et al. 2009).

En el caso de Sympagurus dimorphus su distribución está asociada a aguas circumantárticas, tanto del sur de África como de Sudamérica. Este nuevo registro amplia su distribución hacia el norte, alrededor de $1.000 \mathrm{~km}$ de la cita anterior para Chile, siempre por debajo de los $400 \mathrm{~m}$ de profundidad, asociado a aguas intermedia antárticas que recorren desde el extremo sur hasta el norte de Chile a profundidades entre los 300 a $400 \mathrm{~m}$ de profundidad en el norte y que en el extremo sur es más somera (Silva \& Konow 1975). 


\section{Agradecimientos}

Deseamos dar gracias a la tripulación del Crucero INSPIRE a bordo del B/C Melville (SCRIPPS Institutions). Agradecemos también a los Drs. Andrew Thurber y Lisa Levin de la División integrada de Oceanografía de Scripps Institution of Oceanography (USA), y al Dr. Javier Sellanes de la Universidad Católica del Norte (UCN) por proveer de las facilidades a bordo durante el Crucero INSPIRE. Esta investigación fue auspiciada por el Proyecto FONDECYT No 1100166 (CHILE), y la Integrative Oceanography Division Scripps Institution of Oceanography (USA) y el Ocean Explorer Program from National Oceanic and Atmospheric Administration (NOAA). Nuestros agradecimientos al Dr. Marcos Tavares del Museo de Zoología de la Universidad de São Paulo (MZUSP), por el apoyo y comentarios a este manuscrito.

\section{LITERATURA CITADA}

Cardoso I \& R Lemaitre. 2012. Firts reports of deep-water hermit crabs Parapagurus Smith, 1879 (Decapoda, Parapaguridae) and coelenterate associates from the MidAtlantic ridge and south Atlantic. Crustaceana 85(4-5): 591600.

Guzmán G. 2003. Primer registro para Chile y descripción de la hembra de Cymonomus menziesi Garth, 1971 (Decapoda, Archaeobrachyura, Cymonomidae). Investigaciones Marinas 31(2): 91-94.

Guzmán G \& E Quiroga. 2005. New records of shrimps (Decapoda: Caridea and Dendrobranchiata) in deep waters off Chile. Gayana 69(2): 285-290.

Guzmán G, P Báez \& J Sellanes. 2009. Primer registro de Trichopeltarion corallinus (Faxon, 1893) para el mar de Chile y nuevo registro de T. hystricosus (Garth, en Garth \& Haig, 1971) (Decapoda: Brachyura: Atelecyclidae). Latin American Journal of Aquatic Research 37(2): 275-279.

Haig J. 1955. The Crustacea Anomura of Chile. Reports of the Lund University Chile Expedition 1948-49. Lunds Universitets Ârsskr. N.F. Avd. 2, 51(12): 1-68.

Hebbeln D, WE Arntz, P Böning, H Bohlmann, X Contardo, L Dezileau, TG Ferdelman, S Figueroa, J Fleming, VA Gallardo, D Gerdes, S Giglio, P Gonzales, R Gonzáles, D Gutièrrez, C Hayn, J Kallmeyer, G Klockgether, K Lambers, F Lamy, J Langer, G Lavik, H Löbner, M Marchant, B Meyer-Schack, M Mohtadi, A Montiel, J Niggemann, E Quiroga, OE Romero, M Rondanelli, C Scheurle, F Schewe, RR Schneider, CJ Schubert, J Sellanes, W Serrano, S Stregel, N Teixidó, L Toffin, T Treude, R Villa, C Villagrán, E Walpersdorf, $P$ Wintersteller \& J Wulf. 2001. PUCK: Report and preliminary results of R/V Sonne Cruise SO156, Valparaiso (Chile)-Talcahuano (Chile). March 29-May 14, 2001.
Berichte aus dem Fachbereich Geowissenschaften der Universität Bremen, 182: 1-195.

Henderson JR. 1888. Report on the Anomura collected by H.M.S Challenger during the years 1873-1876. Report on the Scientific Results of the Voyage of H.M.S. Challenger, Zoology 27: 1-221.

Lemaitre R. 1986. Western Atlantic species of the Parapagurus pilosimanus complex (Anomura: Paguroidea: Parapaguridae): Description of a new species and morphological variations. Journal of Crustacean Biology 6(3): 525-542.

Lemaitre R. 1989. Revision of the genus Parapagurus (Anomura: Paguroidea: Parapaguridae), including redescription of the western Atlantic species. Zoologische Verjhandelingen 253: 1-106.

Lemaitre R. 1996. Hermit crabs of the family Parapaguridae (Crustacea: Decapoda: Anomura) from Australia: species of Strobopagurus Lemaitre, 1989, Sympagurus Smith, 1883 and two new genera. Records of the Australian Museum 48: $163-221$.

Lemaitre R. 1998. Revisting Tylaspis anomala Henderson, 1885 (Parapaguriae), with comments on its relationships and evolution. Zoosystema 20(2): 289-305.

Lemaitre R. 1999. Crustacea Decapoda: A review of the species of the genus Parapagurus Smith, 1879 (Parapaguridae) from the Pacific and Indian Oceans. In: Crosnier A (ed). Résultats des Campagnes MUSORSTOM, Vol 20. Mémoirs Muséum National d'Histoire Naturelle, Paris 180: 303-378.

Lemaitre R. 2004. A worldwide review of hermit crab species of the genus Sympagurus Smith, 1883 (Crustacea: Decapoda: Parapaguridae). In: Marshall BA \& B Richer de Forges (eds). Tropical deep-sea benthos. Memoires du Museum National d'Histoire Naturelle 191(23): 85-149.

Lemaitre R \& PA McLaughlin. 1992. Descriptions of megalopa and juveniles of Sympagurus dimorphus (Studer, 1883), with an account of the Parapaguridae (Crustacea: Anomura: Paguroidea) from Antarctic and Subantarctic waters. Journal of Natural History 26: 745-768.

McLaughlin PA, T Komai, R Lemaitre \& DL Rahayu. 2010. Annotated checklist of anomuran decapod crustaceans of the world (exclusive of the Kiwaoidea and families Chirostylidae and Galatheidae of the Galatheoidea) - Part I -Lithodoidea, Lomisoidea and Paguroidea. The Raffles Bulletin of Zoology Supplement 23: 5-107.

Menzies RJ, RY George \& GT Rowe. 1973. Abyssal environment and ecology of the world oceans, $488 \mathrm{pp}$. Wiley Inter-Science, New York.

Parin NV, AN Mironov \& KN Nesis. 1997. Biology of the Nazca and Salas y Gómez submarine ridges, an outpost of the Indo-West Pacific fauna in the eastern Pacific Ocean: composition and distribution of the fauna, its communities and history. Advances in Marine Biology 32: 145-242. 
Porter C. 1906. Materiales para la fauna carcinolojica de Chile. Revista Chilena de Historia Natural 5: 128-138.

Quiroga E, J Sellanes, WE Arntz, D Gerdes, VA Gallardo \& D Hebbeln. 2009. Benthic megafaunal and demersal fish assemblages on the Chilean continental margin: The influence of the oxygen minimum zone on bathymetric distribution. Deep-Sea Research II 56: 1112-1123.

Quiroga E \& LA Levin. 2010. Eunice pennata (Polychaeta: Eunicidae) from active and passive cold seep sites in Central and Southern Chile ( $\left.36^{\circ}-46^{\circ} \mathrm{S}\right)$. Anales del Instituto de la Patagonia 38(2): 31-37.

Retamal MA. 2007. Nota sobre la biodiversidad carcinologica (Stomatopoda y Decapoda) en los fiordos occidentales entre el Guafo y estero Elefantes. Ciencia y Tecnología del Mar 30(1): 149-154.
Saint Laurent M de. 1972. Sur la famille des Parapaguridae Smith, 1882. Description de Typhlopagurus foresti gen. nov., sp. nov., et de quinze espèces ou sous-espèces nouvelles de Parapagurus Smith (Crustacea, Decapoda). Bijdragen tot de Dierkunde 42(2): 97-123.

Silva N \& D Konow. 1975. Contribución al conocimiento de las masas de agua en el Pacífico Sudoriental. Expedición Krill. Crucero 3-4, julio-agosto1974. Revista de la Comisión Permanente del Pacífico Sur 3: 63-75.

Wicksten MK. 1987. Range extensions of offshore decapod crustaceans from California and western Mexico. California Fish and Game 73(1): 54-56.

Zhadan D. 1997. Deep-sea hermit crabs from the submerged ridges Nazca and Sala-y-Gómez, southeastern Pacific (Decapoda Anomura Paguridae). Arthropoda Selecta 6(12): 55-79.

Recibido el 3 de mayo de 2013 y aceptado el 21 de enero de 2014

Editor: Claudia Bustos D. 